\title{
FUNÇÃO EXECUTIVA DE IDOSOS INSTITUCIONALIZADOS E COMUNITÁRIOS: RELAÇÃO COM CAPACIDADES COGNITIVAS E FUNCIONAIS
}

\section{Alessandra Vieira Menezes \\ Alessandra da Silva de Aguiar \\ Elysama Fernandes Alves \\ Layse Biz de Quadros}

Discentes do curso de graduação em Fisioterapia na Universidade Federal de Santa Catarina (UFSC), Brasil.

E-mail: alessandra.v.me@gmail.com

\section{Poliana Penasso Bezerra}

Docente do Curso de graduação em Fisioterapia e Pósgraduação em Ciências da Reabilitação, Departamento de Ciências da Saúde, Centro Araranguá, Universidade Federal de Santa Catarina(UFSC), Brasil.
RESUMO: O objetivo do estudo é caracterizar o perfil demográfico e capacidades cognitivas e funcionais de idosos institucionalizados e não institucionalizados, verificando a associação com a função executiva. A amostra incluiu 25 indivíduos comunitários (20 mulheres; 69,52 $\pm 8,99$ anos) e 26 institucionalizados (11 mulheres; 74,69 $\pm 7,94$ anos; 47,03 $\pm 58,31$ meses de institucionalização). O grupo institucionalizado apresentou escores inferiores nas avaliações cognitivas e funcionais $(\rho<0,001$; teste de Mann-Wihtney). A função executiva, avaliada pela Bateria de Avaliação Frontal, apresentou correlação positiva (teste de Spearman) com Fluência Verbal $(\rho=0,818)$, Mini-exame do Estado Mental $(\rho=0,649)$, Índice de Barthel $(\rho=0,468)$ e Pfeffer $(\rho=-0,70)$ no grupo institucionalizado e apenas com a fluência verbal $(\rho=0,523)$ no grupo comunitário. O maior comprometimento da função executiva no grupo institucionalizado pode estar relacionado à maior dependência funcional e declínio cognitivo observado.

PALAVRAS-CHAVE: Instituição de longa permanência para Idosos; Saúde do idoso institucionalizado; Função executiva; Cognição.

\section{EXECUTIVE FUNCTION OF THE ELDERLY IN INSTITUTIONS: RELATIONSHIP WITH COGNITIVE AND FUNCTIONAL ABILITIES}

ABSTRACT: The demographic profile and cognitive and functional abilities of elderly people in institutions or not are characterized and their association with the executive function verified. Sample comprises 25 elderly people in homes ( 20 females; $69,52 \pm 8,99$ years) and 26 institutionalized (11 females; 74,69 $\pm 7,94$ years; 47,03 $\pm 58,31$ years in the institution). The institutionalized group had lower scores in cognitive and functional assessments $(\rho<0,001$; Mann-Withney Test). The executive function evaluated by the Frontal Assessment Battery showed a positive co-relationship (Spearman's Test with Verbal Fluency $(\rho=0,818)$, Mental State Mini-test $(\rho=0,649)$, Barthel's Index $(\rho=0,468)$ and Pfeffer's Index $(\rho=-0,70)$ in the institutionalized group and only with verbal fluency $(\rho=0,523)$ in the home group. Highest impairment of the executive function in the institutionalized group may be related to greater functional dependence and decreasing cognitive rate.

KEY WORDS: Homes for the Aged; Health of Institutionalized Elderly; Executive function; Cognition. 


\section{INTRODUÇÃO}

Atualmente é crescente o interesse em investigar temas relacionados ao envelhecimento pelo progressivo aumento do número de idosos no mundo (BEZERRA et al., 2012). No Brasil, a população de idosos representa um contingente de quase 19 milhões (IBGE, 2010).

Das estruturas cerebrais, a área do córtex préfrontal é a região mais afetada pelo envelhecimento, repercutindo na função executiva, comprometendo resolução de problemas, manipulação espacial, velocidade mental e identificação de relações complexas entre padrões de estímulos (TURNER; SPRENG, 2012) o que pode influenciar diretamente na capacidade do idoso de conviver em sociedade, seja institucionalizado ou idoso comunitário. Sendo assim, para manter a participação nas atividades cotidianas, o idoso também necessita manter um equilíbrio entre o desempenho cognitivo e a capacidade funcional, preservando a qualidade de vida $\mathrm{e}$ aumentando a autopercepção de bem-estar, por meio da independência funcional e controle do seu ambiente de convívio (COUTLEE; HUETTEL, 2012; FRANCO-MARTÍN et al., 2013).

Sabe-se que a prevalência de comprometimento cognitivo aumenta o potencial de impacto da demência sobre o estado de saúde global influenciando nos cuidados a serem tomados (GODINHO, 2012). Segundo Nakatani et al. (2011), a capacidade funcional compreende a habilidade do indivíduo para desempenhar tarefas no dia-a-dia e viver de forma independente. Essas atividades revelam a capacidade do autocuidado, da execução de seus papéis nas suas tarefas básicas e sociais.

Os declínios executivos podem preceder o início de uma demência em sete a dez anos, sendo importante destacar as funções executivas como marcadoras no diagnóstico diferencial entre demência e envelhecimento fisiológico, tornando relevante sua investigação (ROBINSON et al., 2015).

Sendo assim, o objetivo deste estudo é caracterizar o perfil demográfico e capacidades cognitivas e funcionais de idosos institucionalizados e não institucionalizados, verificando a associação com a função executiva.

\section{METODOLOGIA}

Trata-se de um estudo quantitativo, observacional e transversal, realizado em duas Instituições de Longa Permanência para Idosos (ILPIs) e em idosos da comunidade, frequentadores de um Centro de Referência de Assistência Social (CRAS) destinado a encontros de convivência da terceira idade, situados em associação de municípios do extremo sul catarinense (AMESC).

Os critérios de inclusão estabelecidos foram idosos de ambos os sexos, com idade acima de 60 anos, residentes em instituição ou participantes do CRAS. Os critérios de exclusão estabelecidos foram não concordar em participar da pesquisa, idade inferior a 60 anos, presença de deficiência visual e/ou auditivas graves não corrigidas, dificuldade no desempenho manual por doenças reumáticas ou neurológicas, diagnóstico de distúrbios cognitivos graves e/ou doenças mentais que pudessem impedir o entendimento e a execução dos procedimentos dos testes ou ter pontuação inferior ou igual a 13 pontos no Mini-exame do Estado Mental.

Para caracterizar o perfil dos idosos, analisaramse dados pessoais (idade, sexo), escolaridade (anos de educação formal, ou seja, exercida na escola) e tempo de admissão na instituição (para os idosos institucionalizados). Em seguida, foi aplicado o MEEM para verificação do critério de exclusão proposto. Os idosos que atendiam os critérios de inclusão seguiam no estudo, sendo avaliados por meio dos seguintes testes: Bateria de Avaliação Frontal (BAF), Teste de Fluência Verbal, Escala de Barthel modificada e Índice de Pfeffer.

MEEM é um teste cognitivo breve composto por itens a respeito de orientações espaço temporal, registro, memória de curto prazo, atenção, cálculo, linguagem e praxia constitucional possibilitando uma avaliação concisa do estado mental do paciente. Para idosos utilizam-se os seguintes pontos de corte: (a) analfabeto 13 pontos; (b) baixa escolaridade (1-4 anos)- 13 pontos; (c) media escolaridade (4 a 8 anos) - 18 pontos; (D) alta escolaridade (acima de 8 anos) -26 pontos. (BRUCKI et al., 2003). A BAF tem como objetivo examinar, por meio da aplicação de teste, as funções cognitivas relacionadas ao lobo frontal constituindo de avaliação de conceituação, flexibilidade mental, programação motora, sensibilidade 
e interferência, controle inibitório e autonomia em relação ao ambiente. A pontuação é entre 0 (pior) e 3 (melhor) e o resultado total varia entre 0 e 18 pontos (DUBOIS et al., 2000). Para o teste de fluência verbal (FV), é solicitado ao indivíduo que fale o maior número de palavras (dentro de uma categoria exigida), durante o tempo de $1 \mathrm{~min}$. Neste caso pontuam-se as respostas, mas não se consideram as repetições. Trata-se de um teste simples e capaz de mensurar declínio cognitivo mesmo em fases iniciais, avaliando vários domínios, como memória operacional, linguagem, capacidade de organização e sequenciamento (AMIEVA et al., 2008). O índice de Barthel é um instrumento que avalia o nível de independência do sujeito para a realização de dez atividades básicas de vida: comer, higiene pessoal, uso dos sanitários, tomar banho, vestir e despir, controle de esfíncteres, deambular, transferência da cadeira para a cama, subir e descer escadas. A pontuação da escala varia de 0-100 (com intervalos de 5 pontos), sendo a pontuação mínima de 0 correspondendo à máxima dependência para todas as atividades de vida diárias (AVD's) e a máxima de 100 equivale à independência total (MAHONEY; BARTHEL, 1965). O índice de Pfeffer é constituído por dez itens relacionados com a capacidade do indivíduo para realizar AIVD's (atividades instrumentais de vida diária) e funções cognitivas/sociais como fazer compras, preparar alimentação, manter-se em dia com a atualidade, prestar atenção em programas de rádio, TV e de discuti-los. Para cada questão o idoso pode obter de 0 a 3 pontos, totalizando um máximo de 30 pontos. Uma pontuação maior ou igual a 5 pontos já caracteriza o idoso com comprometimento funcional (PFEFFER et al., 1982).

Por fim, os idosos incluídos na pesquisa foram divididos em dois grupos de acordo com o local de residência, grupo 1 (G1): idosos comunitários e grupo 2 (G2): idosos institucionalizados para verificação de diferenças entre eles em relação ao perfil demográfico, capacidades cognitivas e funcionais e relação da função executiva em cada grupo.

Os dados foram armazenados e analisados com o auxílio do pacote estatístico Statistical Package for Social Sciences (SPSS), versão 21.0. Inicialmente, as variáveis foram analisadas descritivamente por meio de frequências absolutas e relativas (variáveis categóricas) e medidas de tendência central e dispersão (variáveis numéricas). Os critérios paramétricos de normalidade e homogeneidade de variâncias foram analisados pelo teste de normalidade de Shapiro-Wilk. As variáveis numéricas não apresentaram distribuição normal, então, a comparação entre os grupos deu-se pelo teste de MannWihtney para amostras independentes. Correlações de Spearman foram realizadas para testar associações entre as variáveis BAF (medida de função executiva) com as demais avaliações cognitivas e funcionais. A amplitude no resultado do coeficiente varia de -1 (correlação negativa perfeita) a +1 (correlação positiva perfeita), passando por 0 (ausência de correlação). Adotou-se um nível de significância de 5\%.

A pesquisa foi aprovada pelo Comitê de Ética envolvendo Seres Humanos, CAAE 55489216.8.0000.0121 de acordo com o parecer $\mathrm{n}^{0} 1.557 .877$, além de respeitar a resolução $\mathrm{n}^{\circ}$ 466, de 12 de dezembro de 2012.

\section{RESULTADOS}

Da amostra inicial, 51 idosos atenderam aos critérios de inclusão e exclusão propostos, sendo 25 (49\%) comunitários e 26(51\%) institucionalizados (Figura 1). 


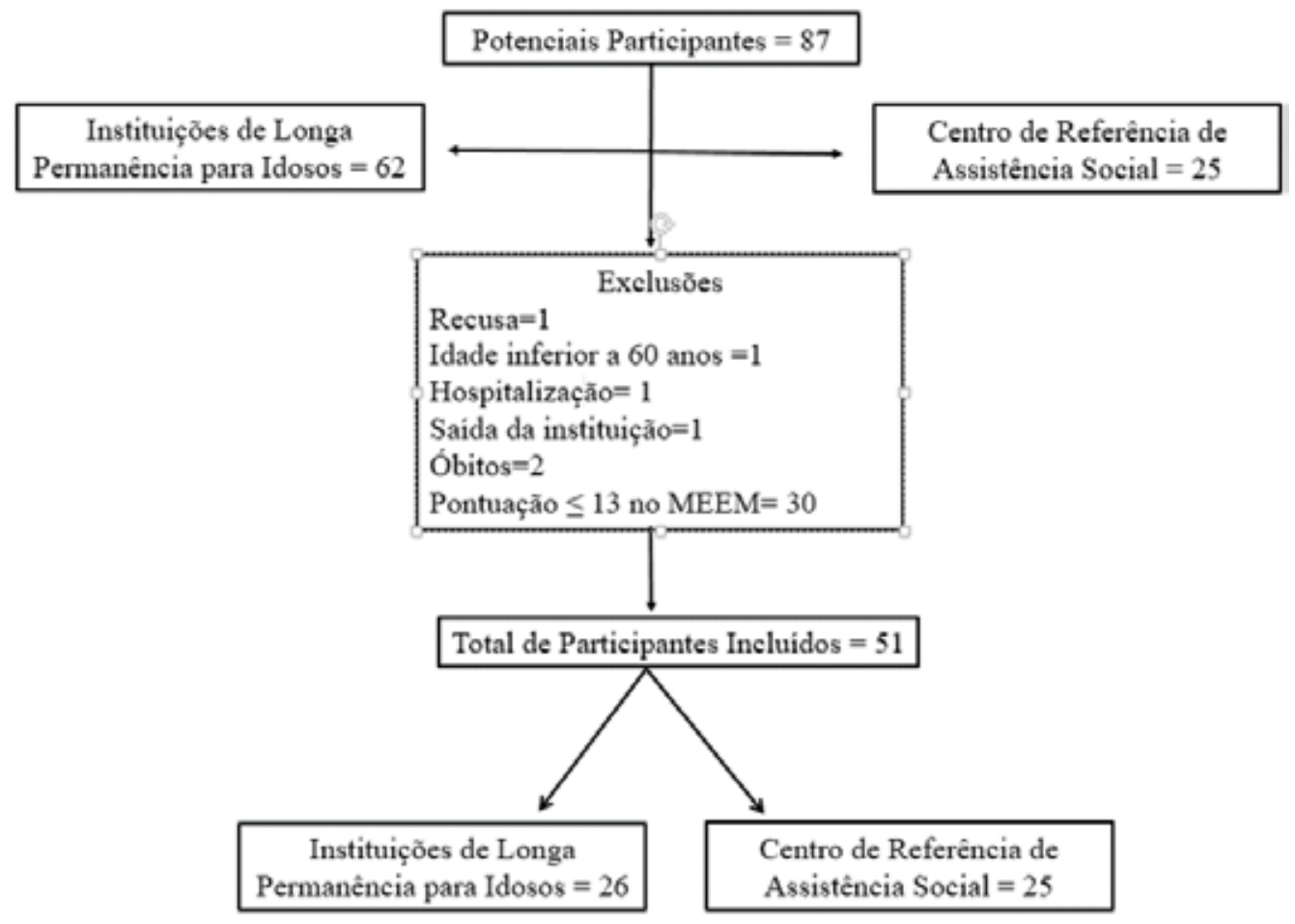

Figura 1. Fluxograma de Inclusões e perdas amostrais

Dos idosos do grupo comunitário, 20(80\%) eram do sexo feminino e cinco (20\%) do sexo masculino, já no grupo institucionalizado $15(57,7 \%)$ eram do sexo masculino e $11(42,3 \%)$ do sexo feminino. O grau de escolaridade do grupo institucionalizado foi de 16 $(61,5 \%)$ que estudaram de um a quatro anos, seis $(23,1 \%)$ que estudaram entre quatro e oito anos e apenas quatro (15,4\%) não eram alfabetizados. Já o grupo comunitário, todos eram alfabetizados, sendo que $17(68 \%)$ estudaram de quatro a oito anos e $8(32 \%)$ que estudaram de um a quatro anos (Figura 2).

\section{G1- comunitário}
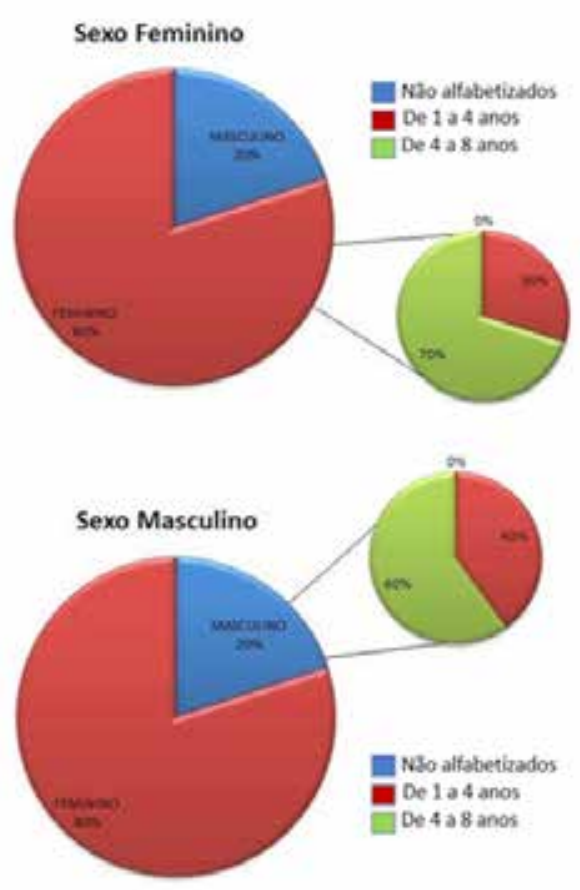
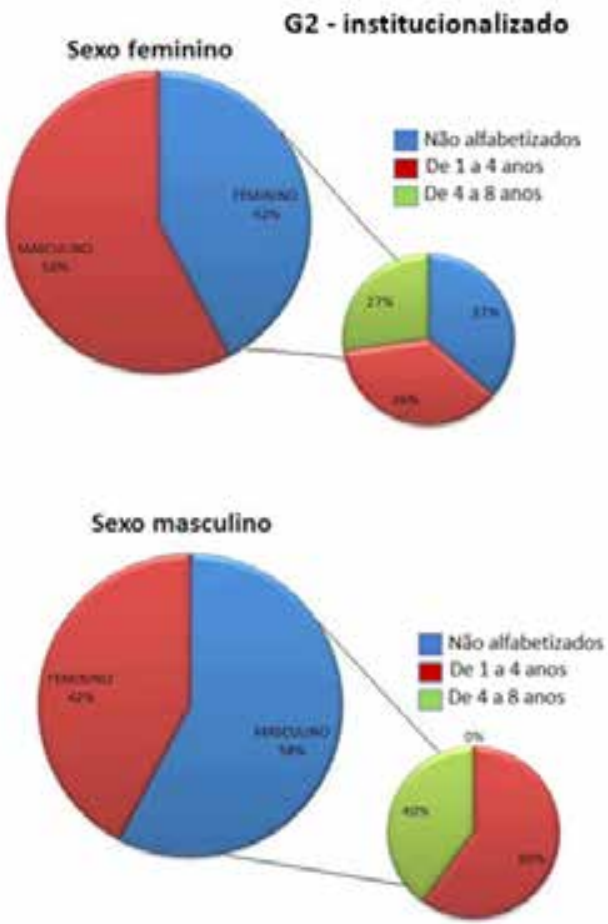

Figura 2. Representação do sexo e grau de escolaridade 
A faixa etária dos participantes variou entre $60 \mathrm{e}$ 96 anos, sendo que o grupo de idosos institucionalizados apresentou média de 47,03 $\pm 58,31$ meses de institucionalização e uma média de idade de 74,69 7,94 anos, sendo superior ao grupo comunitário que alcançou uma média de idade de 69,52 $\pm 8,99$ anos $(\rho=0,007)$.
As avaliações cognitivas e funcionais demonstraram diferença significativa entre os grupos, sendo inferiores no grupo institucionalizado $(\rho \leq 001)$ (Tabela 1).

Tabela 1. Caracterização e comparação entre os grupos em relação à cognição e funcionalidade

\begin{tabular}{|c|c|c|c|c|c|}
\hline & \multicolumn{2}{|c|}{ G1 } & \multicolumn{2}{|c|}{ G2 } & \multirow[b]{2}{*}{$\rho$} \\
\hline & Média $\pm \mathrm{DP}$ & IC (95\%) & Média $\pm \mathrm{DP}$ & IC(95\%) & \\
\hline Idade & $69,52 \pm 8,99$ & $(65,80-73,23)$ & $74,693 \pm 7,94$ & $(71,48-77,90)$ & $0,007^{*}$ \\
\hline Tempo de admissão & 0 & 0 & $47,03 \pm 58,31$ & $(23,48-70,59)$ & $<0,001^{*}$ \\
\hline MEEM & $27,36 \pm 2,51$ & $(26,32-28,39)$ & $20,42 \pm 4,39$ & $(18,64-22,19)$ & $<0,001^{*}$ \\
\hline BAF & $13,64 \pm 2,81$ & $(12,47-14,80)$ & $7,50 \pm 4,33$ & $(5,74-9,25)$ & $<0,001^{*}$ \\
\hline FV & $7,72 \pm 3,37$ & $(6,32-9,11)$ & $3,73 \pm 3,31$ & $(2,39-5,07)$ & $0,001^{*}$ \\
\hline BARTHEL & $97 \pm 14,58$ & $(90,97-103,02)$ & $71,42 \pm 32,89$ & $(58,13-8471)$ & $<0,001^{*}$ \\
\hline PFEFFER & $0,20 \pm 0,81$ & $(-0,13-0,53)$ & $10,07 \pm 8,0$ & $(6,84-13,31)$ & $<0,001^{*}$ \\
\hline
\end{tabular}

Legenda: MEEM: Mini-exame do estado mental; BAF: Bateria de Avaliação Frontal; FV: Fluência Verbal; G1: grupo de idosos comunitários; G2: grupo de idosos institucionalizados; DP: Desvio-padrão; IC: Intervalo de confiança; $\rho$ : nível de significância $(\rho<0,05)$ *Diferença estatisticamente significativa.

A função executiva, avaliada pela Bateria de Avalição Frontal, apresentou correlação positiva com Fluência Verbal $(\rho=0,818)$, Miniexame do Estado Mental $(\rho=0,649)$, Índice de Barthel $(\rho=0,468)$ e Pfeffer $(\rho=-0,70)$ no grupo institucionalizado e apenas com a fluência verbal $(\rho=0,523)$ no grupo comunitário. O maior comprometimento da função executiva no grupo institucionalizado pode estar relacionado à maior dependência funcional e declínio cognitivo observado (Figura 3).
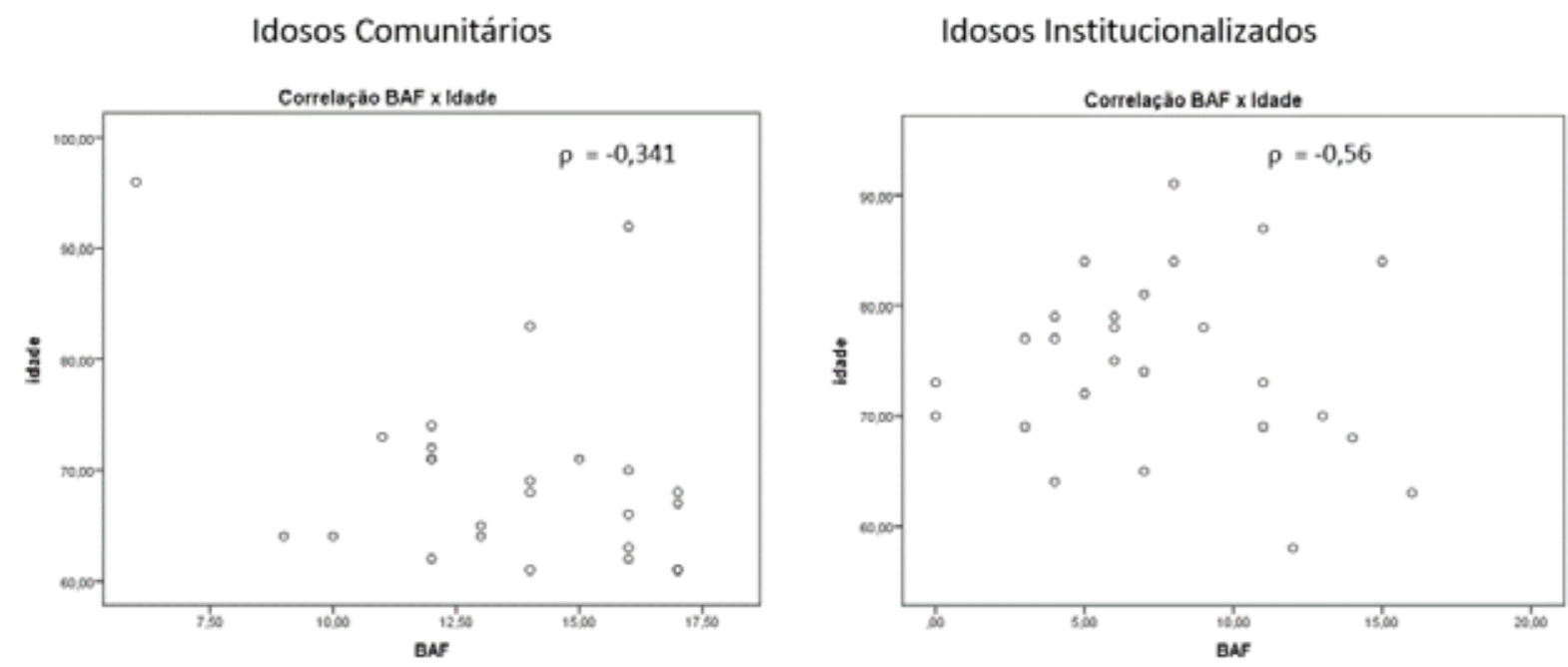

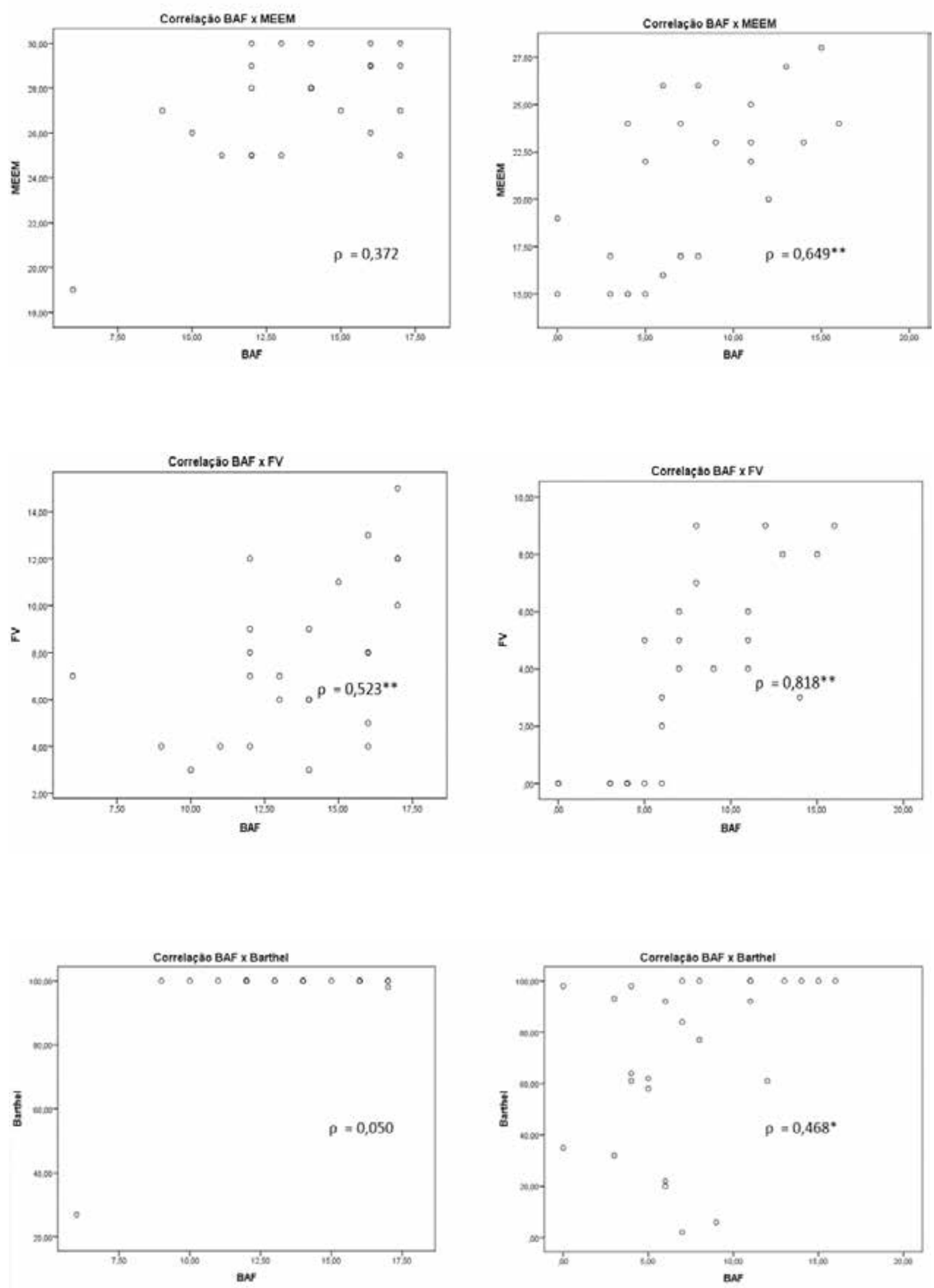

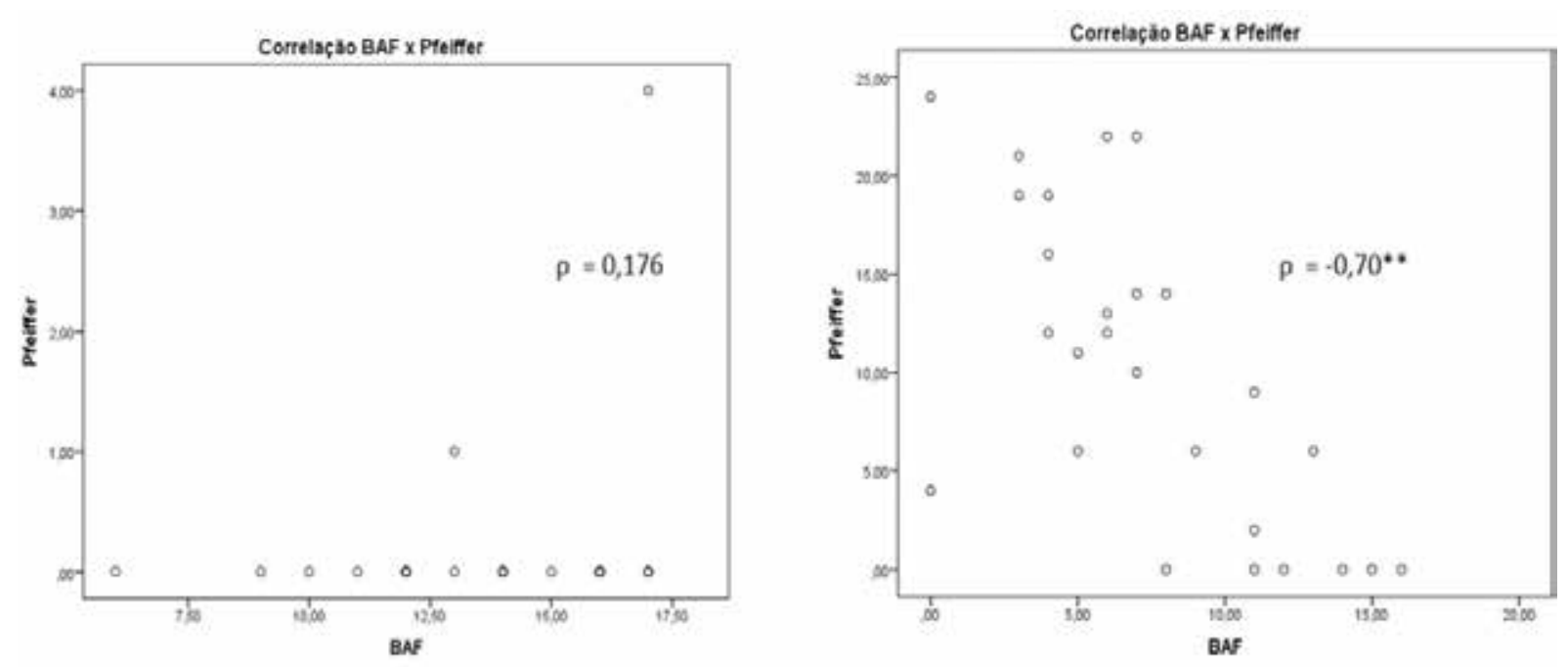

Figura 3. Correlações da função executiva com as variáveis de cognição e funcionalidade Legenda: BAF: Bateria de Avaliação Frontal; MEEM: Miniexame do Estado Mental; FV: Fluência Verbal; Nível de significância: *<0,05 e $* *<0,001$.

\section{DISCUSSÃO}

De acordo com o último censo, as mulheres representavam $55 \%$ do total de idosos no país (IBGE, 2010). No presente estudo podemos constatar que a maioria dos participantes era do sexo feminino, justificado pela maior participação de idosas que frequentam espaços destinados à terceira idade. Entretanto, no universo de idosos institucionalizados, foi observado que a maioria era do sexo masculino, dados que não corroboram com os estudos de Tavares et al. (2015) em idosos institucionalizados, em que a maioria era do sexo feminino. Santos et al. (2010) observaram que tanto idosos comunitários como institucionalizados eram, na sua maioria, do sexo feminino. A institucionalização do idoso de acordo com o sexo se diferencia na literatura atual e pode estar ligada aos contextos social, cultural e econômico de cada região.

Na senescência, o sistema nervoso central é um dos sistemas do organismo mais afetado por alterações nos neurotransmissores e pela hipotrofia cerebral, sendo que estas modificações acontecem preferencialmente nas regiões responsáveis pelas funções cognitivas, como os lobos frontal e temporal, incluindo o sistema límbico (TURNER; SPRENG, 2012; COUTLEE; HUETTEL, 2012). Por esta razão, as funções de alta complexidade como a função executiva são as primeiras capacidades funcionais a serem perdidas com o envelhecimento (BRITO;
AREOSA; LOPES, 2011), apresentando prejuízos mínimos ou significativos da função cognitiva, observados em indivíduos mais velhos (TAYLOR et al., 2012).

No presente estudo, a idade não foi significativamente relacionada à função executiva em nenhum dos grupos participantes. Em contrapartida, Banhado e Nascimento (2007) apontam em seu estudo com idosos que a idade exerceu influência significativa no desempenho da função executiva, sendo que a velocidade de processamento da informação está mais prejudicada em idosos mais velhos. Este mesmo estudo também constatou a relevância do nível de escolaridade, afirmando que a educação formal exercida na escola está relacionada ao melhor desempenho intelectual. No presente estudo não houve comparação da escolaridade pelo fato de os idosos não informarem valores numéricos precisos que permitissem a análise, sendo a informação da escolaridade registrada em categorias. Destaca-se que $15,4 \%$ dos idosos institucionalizados não eram alfabetizados, enquanto que 100\% dos comunitários estudaram pelo menos de um a quatro anos e talvez por esta razão, apresentaram escores melhores na função executiva.

Em relação às capacidades cognitivas e funcionais, houve diferença significativa entre os grupos em todas as variáveis, evidenciando maior declínio das capacidades cognitivas e funcionais no grupo de idosos institucionalizados. Sugere-se que na comunidade, o 
idoso mais debilitado e dependente permanece em sua residência aos cuidados de familiar ou cuidador, não participando de centros de convivência, e por esta razão, não fizeram parte do grupo estudado. Ao passo que em ILP é comum encontrar idosos dependentes, onde o cuidado dentro da instituição é opção da família, isso pode ter influenciado no perfil dos grupos em relação à funcionalidade.

Em recente comparativo de idosas institucionalizadas e participantes de grupos de convivência também pôde ser observado diferença estatisticamente significativa na capacidade cognitiva, em itens específicos como orientação no espaço e no tempo (CARVALHO et al., 2014). Segundo Tavares et al. (2015), o declínio cognitivo, do mesmo modo que as doenças crônicas, significam sérios problemas de saúde pública impactando diretamente na dependência do indivíduo.

Em nosso estudo, percebeu-se forte correlação da função executiva e a cognição no grupo de idosos institucionalizados, ou seja, o declínio cognitivo afeta e é afetado pela tomada de decisões e resoluções de problemas por estar interligados. A cognição se apresenta como uma função cortical e é dividida em subfunções: memória, atenção, orientação, raciocínio, função executiva e organização vísuomotora (FREITAS et al., 2011). De acordo com Soares et al. (2013), o desempenho cognitivo dos idosos está diretamente relacionado à função executiva.

Distúrbios pré-frontais influenciam negativamente tanto as atividades funcionais como as habilidades psicomotoras dos indivíduos (FELIPPE et al., 2014). Entretanto, no presente estudo, foi observado que esta condição importante do comportamento humano, de ser capaz de reagir a uma situação e tomar uma decisão, se mostra moderadamente correlacionada com as atividades básicas de vida diária no grupo de idosos institucionalizados e não significativa em idosos comunitários. Tal condição pode ser explicada pelo fato que atividades rotineiras exijam menos ativação de funções cognitivas frontais do que nas atividades relacionadas ao meio e à capacidade de interagir (MARSHALL et al., 2012). Recente estudo aponta o significado da institucionalização para seu idoso residente, que o relaciona a um lugar para ser cuidado. Entretanto, os autores afirmam que a instituição se torna um lugar ambíguo, pois acolhe, abriga e ao mesmo tempo aprisiona e mortifica. Geralmente as instituições apresentam aspectos singulares, possuindo normas e práticas, condutas e comportamentos próprios. Com isso as atividades são realizadas sob um protocolo de rotinas, sendo iguais para todos, ignoradas as diferenças individuais bem como a história da vida de cada um, consequentemente comprometendo sua identidade e autonomia (OLIVEIRA; ROZENDO, 2014).

Apesar de existirem poucos estudos que confirmem a correlação da função executiva com o desempenho da fluência verbal, neste estudo foi constatada uma forte ligação entre as duas capacidades. Ou seja, o planejamento na execução e a invocação da memória requerida no teste de FV estão muito mais dependentes um do outro, fato constatado em ambos os grupos. Apesar e teste FV ter uma aplicação simples, é considerado uma tarefa cognitiva complexa e muito sensível a todo tipo de dano cerebral, apontando, precocemente, os processos de deterioração executiva (BANHATO; NASCIMENTO, 2007). Deste modo pode-se inferir que os achados de correlação encontrados nesta população de idosos podem também estar relacionados à sua condição de isolamento social ou participação ativa na comunidade e ao grau de dependência.

Apesar da carência de estudos na população de idosos institucionalizados relacionando a função executiva com as habilidades do indivíduo de manter a sua independência e autonomia para fazer compras, cuidar do próprio dinheiro, acompanhar notícias, interagir na sociedade, neste estudo pôde-se perceber a forte interação entre as duas capacidades, no grupo institucionalizado. Nosso estudo sugere que essa forte relação seja relevante para a condição independente dos idosos, ao passo que, dificuldades em realizar essas tarefas cotidianas podem causar isolamento e até mesmo exclusão social (GURE et al., 2013). Carvalho et al. (2014) sugerem que instrumentos de avaliação das atividades básicas talvez não sejam tão sensíveis quanto as escalas que mensurem as atividades instrumentais.

\section{CONCLUSÃO}

Os idosos institucionalizados apresentam declínio significativo nas funções cognitivas e importante dependência nas atividades de vida diária e instrumentais em relação aos idosos comunitários e a função executiva 
está associada ao comprometimento cognitivo e funcional observado.

A função executiva, quando afetada, pode significar o primeiro passo para o comprometimento das demais capacidades, partindo do pressuposto que o indivíduo é inicialmente independente e vive em sociedade. Sendo assim, afirma-se que o ambiente é consequência de sua capacidade de autonomia e autodeterminação. Sabe-se que a incapacidade é limitante física e socioemocional e por esta razão percebeu-se o declínio significativo no grupo de idosos institucionalizados. As funções pré-frontais preservadas são importantes marcadores para detecção de declínios patológicos no envelhecimento.

\section{REFERÊNCIAS}

AMIEVA, H.; GOFF, M. L.; MILLET, X.; ORGOGOZO, J. M.; PÉRÈS, K.; BARBERGER-GATEAU, P.; JACQMIN-GADDA, H.; DARTIGUES, J. F. Prodromal Alzheimer's disease: successive emergence of the clinical symptoms. Ann Neurol., v. 64, n. 5, p. 492-8, 2008.

BANHATO, E. F. C.; NASCIMENTO, E. Função executiva em idosos: um estudo utilizando subtestes da Escala WAIS-III. Psico-USF, v. 12, n. 1, p. 65-73, 2007.

BEZERRA, F. C.; ALMEIDA, M. I.; NÓBREGA-THERRIEN, S. M. Studies on aging in Brazil: literature review. Rev bras geriatr gerontol., vol.15, n.1, p. 155-167, 2012.

BRITO, I. L.; AREOSA, S. C.; LOPES, R. M. Avaliação das Funções Executivas em idosos acometidos por doenças crônica-degenerativas. In: JORNADAS DE PESQUISA EM PSICOLOGIA - DESAFIOS ATUAIS NAS PRÁTICAS DE PSICOLOGIA, 4., 2011. Anais... Santa Cruz do Sul: UNISC, 2011. p. 51-66.

BRUCKI, S. M. D.; NITRINI, R.; CARAMELLI, P.; BERTOLUCCI, P.H.R.; OKAMOTO, I.H. Sugestões para o uso do mini-exame do estado mental no Brasil. Arq Neuropsiquiatr., v. 61, n. 3, p. 777-781, 2003.

CARVALHO, V. L. D.; MEDEIROS, D. V. D. C.; SILVA, J. C. A.; BARBOSA, S. G.; SOUZA, L. L. D. Comparação da função cognitiva de idosas institucionalizadas e das participantes de centro de convivência. RBCEH, Rev. bras. ciênc. envelhecimento hum., v. 11, n. 1, p. 46-53, 2014.

COUTLEE, C. G.; HUETTEL, S. A. The functional neuroanatomy of decision making: prefrontal control of thought and action. Brain Res., v. 1428, p. 3-12, 2012.

DUBOIS, B.; SLACHEVSKY, A.; LITVAN, I.; PILLON, B. The FAB: A Frontal Assessment Battery at bed side. Rev Neurol (Paris), v. 55, n. 11, p. 1621-1626, 2000.

FELIPPE, L. A.; OLIVEIRA, R. T. D.; GARCIA, M.; SILVAHAMU, T. C. D. D.; SANTOS, S. M. S.; CHRISTOFOLETTI, $G$. Executive functions, activities of daily living and motor skill of elderly people with neurodegenerative diseases. J. Bras. Psiquiatr., v. 63, n. 1, p. 39-47, 2014.

FRANCO-MARTÍN, M.; PARRA-VIDALES, E.; GONZÁLEZPALAU, F.; BERNATE-NAVARRO, M.; SOLIS, A. Influencia del ejercicio físico en la prevención del deterioro cognitivo en las personas mayores: revisión sistemática. Rev Neurol., v. 56, n. 11, p. 545-554, 2013.

FREITAS, V.; PY, L. E. Tratado de Geriatria e Gerontologia. 3. ed. Rio de Janeiro: Guanabara Koogan, 2011. ISBN 8527719053.

GODINHO, C. C. Incidência de demência e comprometimento cognitivo leve e identificação de preditores numa amostra de base populacional. 2012. 87f. Tese (Doutorado em Ciências Médicas) Universidade Federal do Rio Grande do Sul, Porto Alegre, 2012.

GURE, T. R.; LANGA, K. M.; FISHER, G. G.; PIETTE, J. D.; PLASSMAN, B. L. Functional limitations in older adults who have cognitive impairment without dementia. J Geriatr Pshyciatry Neurol., v. 26, n. 2, p. 78-85, 2013.

Instituto Brasileiro de Geografia e Estatística. Censo demográfico 2010: características da população e dos domicílios: resultados do universo. Rio de Janeiro, IBGE, 2010.

MAHONEY, F. I.; BARTHEL, D. W. Functional evaluation: 
the Bathel Index. Md. State Med. J., v.14, p. 56-61, 1965.

MARSHALL, G. A.; AMARIGLIO, R. E.; SPERLING, R. A.; RENTZ, D. M. Activities of daily living: where do they fit in the diagnosis of Alzheimer's disease? Neurodegener Dis Manag, v. 2, n. 5, p. 483-91, 2012.

NAKATANI, A. Y. K.; SILVA LB, BACHION MM, NUNES DP. Capacidade funcional de idosos na comunidade e proposta de intervenção pela equipe da saúde. Rev Eletrônica Enferm., v. 11, n. 1, p. 144-50, 2009.

OLIVEIRA, J. M. D.; ROZENDO, C. A. Instituição de longa permanência para idosos: um lugar de cuidado para quem não tem opção? Rev Bras Enferm., v. 67, n. 5, p. 773-9, 2014.

PFEFFER, R. I.; KUROSAKI, T. T.; HARRAH, C. H.; CHANCE, J. M.; FILOS, S. Measurement of functional activities in older adults in the community. J Gerontol., v. 37, n. 3, p. 323-329, 1982.

ROBINSON, L.; TANG, E.; TAYLOR, J. P. Dementia: timely diagnosis and early intervention. BMJ., p. 1-6, 2015.

SANTOS, C. S.; CERCHIARI, E. A. N.; ALVARENGA, M. R. M.; FACCENDA, O.; OLIVEIRA, M. A. D. C. Avaliação da confiabilidade do mini-exame do estado mental em idosos e associação com variáveis sociodemográficas. Cogitare Enferm., v. 15, n. 3, p. 406-412, 2010.

SANTOS, S. M. S.; CHRISTOFOLETTI, G. Executive functions, activities of daily living and motor skill of elderly people with neurodegenerative diseases. J. Bras. Psiquiatr., v. 63, n. 1, p. 39-47, 2014.

SOARES, R. M.; DINIZ, A. B.; CATTUZZO, M. T. Associação entre atividade física, aptidão física e desempenho cognitivo em idosos. Motricidade, v. 9, n. 2, p. 85-94, 2013.

TAVARES, P. D. N.; SCHMIDT, J. H.; WITTER, C. Efeitos de um programa de intervenção no desempenho cognitivo e sintomatologia depressiva em idosos institucionalizados. Rev. kairós, v. 18, n. 2, p. 103-123, 2015.

TAYLOR, M.E.; KETELS, M.M.; DELBAERE, K.; LORD, S. R.;
MIKOLAIZAK, A. S.; CLOSE, J. C. T. Gait impairment and falls in cognitively impaired older adults: an explanatory model of sensorimotor and neuropsychological mediators. Age Ageing, v. 41, n. 5, p. 665-9, 2012.

TURNER, G. R.; SPRENG, R. N. Executive functions and neurocognitie aging: dissociable patterns of brain activity. Neurobiology of Aging, v. 33, n. 4, p. 826.e1-826.e13, 2012.

Recebido em: 16 de agosto de 2016 Aceito em: 08 de dezembro de 2016 